\title{
Relationship of body image and emotion regulation strategies among female students
}

\author{
Kourosh Mohammadi Hasel ${ }^{1^{*}}$, Maryam Didehdar Ardebil ${ }^{2}$ \\ ${ }^{1}$ Tehran nonprofit institute of higher education, Tehran, Iran \\ Email: kolo950@gmail.com \\ ${ }^{2}$ Panjab University, Chandigarh, India \\ Email:kolo950@gmail.com \\ *Corresponding author
}

\begin{abstract}
Physic and appearance is very important in teenage period on the other hand they are not skilled enough in case of adaptive strategies. The aim of this study was to investigate the relationship between worries and cognitive emotional strategies with body image. In this correlational study, 210 students from all of high school students was selected as sample and completed standard body image questionnaire, cognitive emotional regulation strategies and worries questionnaire. The collected data were analyzed using software, mean, standard deviation, Pearson correlation and regression. The findings indicated that there was a positive and direct correlation between physical anxiety and physical activity, while between positive emotional cognitive strategies and body image, there was a significant negative correlation. The results of regression analysis also indicated that strategies for cognitive emotional regulation and worry play a role in predicting the physical image. These findings highlight the importance of paying attention to the psychological state of adolescents at this stage of life, indicating that excessive lack of skill and concern can cause irreparable mental harm in these adolescents and have a negative effect on their progress.
\end{abstract}

Keywords: emotion regulation, body image, teen

\section{Introduction}

Adolescent's age involved with social stress, such as identity, university exam and job prospects. The stress of these changes can impact the health (Firoozi et al., 2012). On the other hand, teenage stage are identified as physical and psychological and social changes. Researches showed most of teens are not satisfied with their body shape. They 
often spends hours in front of the mirror and pay attention to their dressing. Body dysmorphic disorder appears to be more likely to occur in response to physical and hormonal changes during puberty (Habib Allah and Soltanizadeh, 1394).

The fact is that body image, for teens is very important (how to see our size, shape, weight, face, movement, and actions), there is also an attitude that relates to how we feel about (Amani, 1391). Anxiety can exacerbate this feeling in a person, and worrying about others idea can cause a permanent problem for a person (Gilbert, 2005). Borozkowski et al. (2005) argue that adolescents are very concerned about the evaluation and have many concerns (Mohammadi and Sajjadinejad, 2007).

Gilba-Scotsman et al. (2006) found that females who do not have a proper image of their own body, have less awareness of their emotions compared with the healthy group. Moradian (2014) also found that students who applied for cosmetic surgery received lower scores in emotional processing. Naturally, adolescents have their own concerns, but the persistence of these concerns can make a lot of trouble. However, factors such as emotional regulation strategies that an individual uses to counteract stress can modify or exacerbate the impact of this concern (Hosni et al., 1395).

Emotion regulation strategies are associated with the reduction of negative emotions and emotional management. These strategies help a person to choose the best decision in cognitive and emotional coping in different situations (Steartur et al., 2014). Emotion regulation strategies not only focus on negative emotions, but also on positive emotions, they classified into two adaptive categories (positive focus, positive reassessment, refocus planning, acceptance, Maladaptation (blame, disaster, rumination, blame for others). Ineffective emotional regulation is one of the main attributes of anxiety and anxiety disorders (Rybaby et al., 2012).

Nejati et al. (2014) showed that people with a negative body image have difficulty choosing emotional strategies, and they are often incapable of setting up emotions in unpleasant situations. Hughes and Gulen (2011) also reported that 533 adolescents aged 20 to 11 years old reported that emotional regulation moderates depression among those who are self-mediated, but are not effective in symptoms associated with anxiety and anxiety. Coley et al. (2012) also found that there was a relationship between body image problems and conflicting strategies such as anxiety and anxiety. The review of study showed no local study have been conducted in this area; Therefore, in this study, attempts to answer this question whether emotional regulation strategies and body image concerns are related to body image of adolescent females. 


\section{Material and Methods}

This study was a descriptive- correlational study. The research population consisted all the final year students studying in the high schools in non-profit high schools which included 2 girls' high schools. The number of female students enrolled in the third grade high school in the time period of the research was about 460, which was the population of the present study. The sample size was estimated to be 210 according Morgan sampling table.

Cluster sampling was performed. Clusters included three levels (1) girls or boys, (2) school years, and (3) classes. In fact, one kind of school was selected randomly from four types of schools (state), a girl or boy by coin throwing, a school year by simple random method, and classes were randomly selected from classes in the third grade of a class.

Data collected by two questionnaire: Cognitive Emotion Regulation Questionnaire (CERQ): This questionnaire was developed by Garnefski, et al. In 2001 in the Netherlands. The questionnaire has 36 items. This scale consists of 9 sub-scales, each of which assesses a specific strategy. The subcategories are: blaming oneself, blaming others, accepting events, revising planning for how to deal with an event, re-focusing positively on pleasurable issues rather than thinking about that actual occurrence, rumination, re-evaluating positively, reaching an outline (Thoughts about the relative occurrence of the event compared with other events) and catastrophic. The total score scores range is from 36 to 180 .

Validity and reliability of the Persian version of CERQ-P: there was a good internal consistency (Cronbach's alpha was 0.76 to 0.92). The female scores and total score of the subscales were significantly correlated $(r=0.46$ to $r=0.75)$, and the value of the coefficients of re-test correlation (from 0.51 to 0.77 ) indicated the stability of the scale. The main component analysis supported the 9-factor model of the emotional cognitive ordering questionnaire, which explained $74 \%$ of variance. The degree of internal relations between subscales was relatively high (0.22 to 0.67). (Hosni, 1389). Cronbach's alpha in this study was obtained for a total score of 0.80 .

-Body image Questionnaire: The Mendelson et al. (2001) questionnaire of trust and satisfaction from the body, which has a 23 questions with a Likert scale of 5 degrees from "totally disagree" with a score of zero to "fully agrees" with a score of 5 . The questionnaire shows a general and responsive view of its appearance and body, and its score ranges from zero to 115 , with a higher score indicating greater satisfaction with 
the body. A score of less than $50 \%$ of the total score is considered as a weak body impression, the score of $75 \%$ is equivalent to a satisfactory average body and above $75 \%$ as a good body satisfaction. Differential validity of this scale indicates the separation power of individuals in both upper and lower groups. The reliability of this scale was obtained by a re-test. The correlation coefficient between two runs was 0.71 (Omidvaro et al., 2002). The credibility of this scale in Iran was obtained by Cronbach's alpha (0.91) (Taheri Tarbet et al., 2013). The Cronbach alpha coefficient of this questionnaire was 0.77 .

After collecting data statistical analysis conducted with SPSS version 20 to analyze the data. Descriptive statistics such as mean, standard deviation, as well as inferential statistics, Pearson correlation coefficient and regression were used. In addition, Kolmogorov-Smirnov was used to evaluate the distribution.

\section{Results}

The mean scores of participants in the emotional regulation strategies variable were 87.34 and standard deviation was 12.212, in the body image variable 47.92 and standard deviation 5.92, respectively. The results of the study of the role of cognitive emotional regulation and concern in predicting body image on stepwise regression are presented below.

Table-1- The Role of Emotion regulation Strategies of body image Prediction of Participants

\begin{tabular}{rrrrr}
\hline $\mathrm{p}$ & $\mathrm{Se}$ & $\mathrm{R} 2$ & $\mathrm{R}$ & Model \\
\hline $0 / 000$ & $3 / 59$ & $0 / 59$ & $0 / 77$ & $* 1$ \\
\hline $0 / 000$ & $3 / 42$ & $0 / 67$ & $0 / 82$ & $* * 2$ \\
\hline
\end{tabular}

As can be seen in the table, negative emotion regulation strategies alone can predict $59 \%$ of the variation of body image $(\mathrm{P}=0.000)$, but when the variable of positive emotional adjustment strategies is also included, this figure is $67 \%$.

\section{Conclusion}

The findings showed that emotional regulation strategies are predictive of body image in female adolescents. The result of the regression shows that emotion regulation strategies explains $67 \%$ of body image variance. These results are consistent with the findings of Goli et al. (2012); Asgari et al. (2009); Burdley et al. (2015); Davoudi et al. (2016). 
In explaining this finding, it can be said that the correlation between body image and negative thoughts is so strong that their differentiation in some studies is not so simple. Certainly people who are more anxious are more likely to feel that they do not look good. Even the anxiety and physical impairment problems have been identified in the background, but there are still differences as to whether anxiety and anxiety lead to physical impairment or vice versa is a physical impairment that will cause anxiety disorder in the future (Wichanson, 2008). Due to the fact that adjustment of emotion helps to regulate emotional processes, a disorder in the management of excitement leads to inadequacies in stimulating emotions and emotions, which in turn leads to the inability to control the negative emotions, which in turn can lead to the creation or maintenance of several disorders

People are often concerned about being evaluated by others, and one of these evaluations, which is very important during adolescence, is to assess their appearance (Gilbert and Meyer, 2005). The persistence of these thoughts in people who are very worried about leads to many disruptions and increases personal concern (Seraj Khorrami, 2016). On the other hand, people who are having trouble adjusting their emotions have different emotions in different situations, and are more likely to be anxious and worried. These people may be more sensitive to the image they are and more likely to be more responsive to others (Rabie et al., 2012).

When an individual experiences a negative evaluation, he is more likely to avoid others and a negative body image of his body forms and acts like a schema. The special trigger events, such as situations where a person has to expose his or her body to others, activates these schemas. In turn, this in turn leads to the care of the negative interpretation of the behavior of others, the avoidance behavior, the attempt to cover and conceal his body, reassurance and remedial action. These behaviors provide a source of negative emotional and emotional experiences about the body and concern about this. This issue causes persistence and deterioration of concern and creates conditions for the formation of mental disorders (Glyan Tehrani et al., 2014), which can result in undesirable effects in different ways.

In general, the findings of this study showed that the regulation of emotional strategies and, can predict changes in the body image. As previous review of previous research showed that body image problems in adolescents lead to various disorders, disrupt their development and affect the future of adolescents and may even lead to suicide; on the other hand, a high level of anxiety among adolescents On the other hand, low awareness about strategies for adjusting excitement leads to more problems and causes more 
problems. Therefore, it is important to pay attention to the related variables and designing the necessary education for adolescents. It is also suggested that in subsequent research students of different age groups compare the variables of emotion regulation strategy, physical picture and level of concern, and in these comparisons the social and economic class and sex are also included.

\section{References}

Amani A (2012). Comparison of emotional anxiety and body image of obese, overweight and normal people. Master's dissertation of psychology, Shiraz University.

Asgari P, Pasha Gha, Aminian M. (2009). Relationship between emotional regulation, psychological stresses of life and body image with eating disorders in women. Thought and Behavior (Applied Psychology) 4 (13): 65-78

Borjali, A., Sohrabi, F., \& Farrokhi, N. A. F. (2015). The Role of Emotion Regulation Difficulties as a Mediator of the Relationship between Body Image Disturbance and Disordered Eating Behavior. International Journal of Pediatrics, 3(2.1), 23-32.

Dasheiri Gha, Golsari M, Borjali A., Sohrabi F. (2009). Psychometric Properties of the Persian Version of the Pennsylvania Statewide Worries Questionnaire in Students. Journal of Clinical Psychology, 1, 4. 67-75.

Davodi, I., Firoozi, A. A., \& Zargar, Y. (2016). The Relationship Between Symptoms of Eating Disorders and Worry About Body Image, Attachment Styles, and Cognitive Emotion Regulation Strategies Among Students of Ahvaz Jundishapur University of Medical Sciences. Jentashapir Journal of Health Research, 7(1).

Firoozi, AS (2012). Investigating the relationship between attachment styles, cognitive strategies of emotion regulation and anxiety about body image with signs of eating disorders in students of Jundishapur University of Medical Sciences, Ahvaz. Master thesis, Jundishapur University of Ahvaz

Gilbert, N. \& Meyer, C. (2005). Fear of Negative Evaluation and the Development of Eating Psychopathology: A Longitudinal Study Among Nonclinical Women. International Journal of Eating Disorder. 37, 307-312.

Gilboa - schechtman, E., Avnon, L., Zubery, E., and Jeczmien, P. (2006).Emotional processing in eating disorders: specific impairment orgeneral distress related deficiency? Journal of Depression and Anxiety,23(6), 331-339. 
Gleyan Tehrani Sh, Ghayasvand M, Mir Mohammad Ali M., Mehran A. (2014). Body image of obese teenage girls in Tehran with depression, anxiety and tension. Monitor: 13,$4 ; 433-440$.

Habibollahi, A., Soltanizadeh (2015). Effectiveness of admission and commitment therapy on physical dissatisfaction and fear of negative evaluation in adolescent girls with abdominal disorder. Journal of Mazandaran University of Medical Sciences . 25 (134): 278-290

Hasani C, Ariana K (2016). Emotional cognitive regulation strategies, anxiety and impulsivity in bipolar disorder with and without comorbidity with obsessive-compulsive disorder. Iranian Journal of Psychiatry and Clinical Psychology. 1395; 22 (1): 39-49

Hosni, c. (2010). Psychometric properties of emotional cognitive regulation questionnaire. Journal of Clinical Psychology, 3, 83-73.

Hughes, E. K., \& Gullone, E. (2011). Emotion regulation moderates relationships between body image concerns and psychological symptomatology. Body Image, 8(3), 224-231.

Kollei, I., Brunhoeber, S., Rauh, E., de Zwaan, M., \& Martin, A. (2012). Body image, emotions and thought control strategies in body dysmorphic disorder compared to eating disorders and healthy controls. Journal of Psychosomatic Research, 72(4), 321-327.

Mohammadi N, Sajjadinejad M. (2007). The relationship between physical impairment, fear of negative evaluation and self-esteem with social anxiety. New Psychological Research (Psychology, University of Tabriz) 2 (5); 55-65

Moradian, F. (2014). Emotional processing between students applying for beauty. Thesis . Islamic Azad University of Karaj Branch

Nejati, S., Rafienia, P., Sabahi, P., \& Rajezi Esfehani, S. (2013). The comparison of Emotion regulation strategies in obese women with negative and positive body image. Iranian Journal Of Cognition And Education, 1(1), 1-6.

Omidvar, N., Eghtesadi, S., Ghazi-Tabatabaei, M.,Minaei, S., Samareh, S. (2002). Body Image and ItsRelation with BMI and Food Use Pattern amongAdolescents in Tehran Research in Medicine.Research in Medicine, 26(4), 257-264.

Sattarpour F, Ahmadi A., Bafandeh Qaramalkhi H. (2014). The Effectiveness of Exercise Therapy Training Reduces Depression Symptoms Of Students. Health education and health promotion 1 (1): 31-38 
Seraj khorrami, zadehsan z (2016). Correlation between Physical Image Concerns, Early Maladaptive Schemes and Social Anxiety Scale among Female Students of Islamic Azad University. Journal of Social Psychology, 10 (38): 80-68.

Taheri Belaghan Torbatyah, Holy Quran, Primordial Abdulm (2013). Student Psychometric Psychometric Specification Scale of Image Satisfaction. Journal of Behavioral Sciences. 11: 522-531.

Wichianson, J. R., Bughi, S. A., Unger, J.B (2008). Perceived stress, Coping and nighteating in college students. Institute for HealthPromotion and Disease prevention Research, University of SouthernCalifornia Keck school of Medicine, Los Angeles, CA, USA. 